\title{
Robust Adaptive Exponential Synchronization of Two Different Stochastic Perturbed Chaotic Systems with Structural Perturbations
}

\author{
A. Soup Tewa Kammogne and H. B. Fotsin \\ Laboratory of Electronics and Signal Processing, Department of Physics, Faculty of Science, University of Dschang, P.O. Box 67, \\ Dschang, Cameroon \\ Correspondence should be addressed to A. Soup Tewa Kammogne; kouaneteoua@yahoo.fr
}

Received 26 May 2015; Revised 31 August 2015; Accepted 2 September 2015

Academic Editor: Shuli Sun

Copyright (c) 2015 A. S. T. Kammogne and H. B. Fotsin. This is an open access article distributed under the Creative Commons Attribution License, which permits unrestricted use, distribution, and reproduction in any medium, provided the original work is properly cited.

\begin{abstract}
The robust adaptive exponential synchronization problem of stochastic chaotic systems with structural perturbations is investigated in mean square. The stochastic disturbances are assumed to be Brownian motions that act on the slave system and the normbounded uncertainties exist in all parameters after decoupling. The stochastic disturbances could reflect more realistic dynamical behaviors of the coupled chaotic system presented within a noisy environment. By using a combination of the Lyapunov functional method, the robust analysis tool, the stochastic analysis techniques, and adaptive control laws, we derive several sufficient conditions that ensure the coupled chaotic systems to be robustly exponentially synchronized in the mean square for all admissible parameter uncertainties. This approach cannot only make the outputs of both master and slave systems reach $H_{\infty}$ synchronization with the passage of time between both systems but also attenuate the effects of the perturbation on the overall error system to a prescribed level. The main results are shown to be general enough to cover many existing ones reported in the literature.
\end{abstract}

\section{Introduction}

Synchronization is a fundamental phenomenon that enables coherent behavior in coupled dynamical systems. Since Pecora and Carroll [1] proposed a method to synchronize two identical chaotic systems with differential initial conditions, chaos synchronization has attracted much attention from various fields during the last decades $[2,3]$. Many methods and experimental techniques have been presented to realize the synchronization of two identical or different chaotic systems [4], such as adaptive control [5], sliding mode control $[6,7]$, nonlinear feedback control $[8,9]$, and fuzzy system based control [10]. Among all these methods, adaptive control method has been used widely to treat the unknown parameters and uncertainties [11, 12]. For example, a strategy for adaptive synchronization of some electrical chaotic circuit based nonlinear control and robust adaptive generalized projective synchronization of GenesioTesi chaotic system with uncertain parameters are discussed in $[13,14]$, respectively. In [15], the stability of adaptive synchronization of chaotic systems was proposed by Sorrentino et al. A robust adaptive synchronization for a class of uncertain chaotic systems with application to Chua's circuit was presented by Koofigar et al. [16]. Hu et al. developed a robust adaptive finite-time synchronization of two different chaotic systems with parameter uncertainties [17]. Bowong and Tewa investigated the unknown inputs' adaptive observer for a class of chaotic systems with uncertainties [18]. However, we have noted that, in all of the above mentioned papers, the chaotic systems are deterministic differential equations without any random parameters. In addition, chaos synchronization is far from being straightforward because different aspects affect significantly the ability of systems to synchronize. A particularly important aspect is to consider the presence of many disturbances and uncertainties sources, which are unavoidable parts of any practical implementation. Therefore, robustness is a very desirable characteristic of a synchronization approach. In general, it is worth pointing out that the stochastic factors like uncertainty of the structure parameter, perturbation of external noise, and stochastic 
input are ubiquitous in nature, society, economy, and realistic engineering. Under normal circumstances, those stochastic factors just play a minor influence. However, when the designer of the system needs to make a choice, it will become a dominant factor which could affect the trend of deterministic system [19].

Synchronization of chaotic systems affected by both structural and stochastic disturbances poses new challenges for the understanding of stability, sensitivity and robustness, bifurcations and chaos, and so forth. When analyzing the dynamical behaviors of chaotic systems, stochastic disturbances and modeling errors are probably two of the main sources that result in uncertainties. To overcome these difficulties, various adaptive synchronization schemes have been proposed and investigated (see an excellent text in [20]). An adaptive almost surely asymptotically synchronization for stochastic delayed neural networks with Markovian switching was discussed by Ding et al. [21] while Li and $\mathrm{Fu}$ proposed the synchronization of chaotic delayed neural networks with impulsive and stochastic perturbations [22]. M. Chen and W.-H. Chen proposed robust adaptive neural network synchronization controller design for a class of time delay uncertain chaotic systems in [23] and a robust stability and $H_{\infty}$-control of uncertain systems with impulsive perturbations [24]. Recently, Fang et al. developed a robust adaptive exponential synchronization of stochastic perturbed chaotic delayed neural networks with parametric uncertainties [25]. It appears clearly from the above works that most did not consider structural perturbations. Some previous research such as $[24,25]$ took into account the structured uncertainties with the stochastic perturbations but supposed that the parametric uncertainties are time varying and norm-bounded and satisfy certain elementary conditions such that the uncertain matrices can be decomposed in product of Lebesque measurable function in order to develop the problem in terms of linear matrix inequality. However, this assumption seems bulky in engineering applications and also leads to the unpredictability of the LMI. According to the best of our knowledge, there are still few results about the synchronization of chaotic systems with both structural perturbations and stochastic disturbances. Therefore, robust adaptive synchronization analysis for uncertain stochastic systems has emerged as a challenging research issue.

In this paper, we discuss the asymptotical synchronization and almost surely synchronization for two different chaotic systems with the consideration of both structural and stochastic perturbations. With the help from the Lyapunov functional method and adaption method, we employ the robust analysis tool and the stochastic analysis techniques to derive some relevant conditions under which the coupled systems is globally robustly synchronized in the mean square for all admissible parameter uncertainties. These conditions guarantee the robustness of the controller against the effect of exoteric perturbations. The rest of the paper is organized as follows: Section 2 presents the Problem's formulation and preliminaries. In Section 3 we present our main result that consists of a new controller that is robust enough against the effect of exoteric perturbation of modified Colpitts oscillator and Chua's circuit. The computer simulation is given in Section 4. Finally, conclusions are presented in Section 5.

\section{Problem's Formulation and Preliminaries}

Let two classes of nonlinear systems be given in the following form:

$$
\begin{aligned}
d x(\tau) & =(A x(\tau)+D \varphi(x(\tau))+B) d \tau, \\
y(\tau) & =C x(\tau) \\
d \hat{x}(\tau) & =(F \hat{x}(\tau)+G f(\widehat{x}(\tau))) d \tau, \\
\tilde{y}(\tau) & =C \widehat{x}(\tau),
\end{aligned}
$$

where $x \in R^{n}$ and $\hat{x} \in R^{n}$ are the systems states and $y \in$ $R^{n \times 1}$ and $\tilde{y} \in R^{n \times 1}$ are the output vectors of systems (1) and (2), respectively. $A, F, D, G, C \in R^{n \times n} ; B \in R^{n \times 1}$ are known matrices such that $(A, C)$ and $(F, C)$ are observable. $(\varphi, f)$ : $R^{n} \rightarrow R^{n}$ are nonlinear continuously differentiable vector function.

Considering the effect of structural and exoteric perturbation on system's parameters, the master system is given by

$$
\begin{aligned}
& d x(\tau) \\
& \quad=((A+\Delta A(\tau)) x(\tau)+D \varphi(x(\tau))+B+\Delta B(\tau)) d \tau, \\
& y(\tau)=C x(\tau) .
\end{aligned}
$$

Based on the observer method, the slave is constructed with nonidentical parametric uncertainties and stochastic perturbation given by

$$
\begin{aligned}
d \widehat{x}(\tau)=[ & (F+\Delta F(\tau)) \hat{x}(\tau)+G f(\widehat{x}(\tau))+u(\tau)] d \tau \\
& +\sigma(t, x(\tau)-\widehat{x}(\tau)) d \widetilde{\omega}(\tau),
\end{aligned}
$$

where $\Delta A(\tau)$ and $\Delta F(\tau)$ are bounded structural variations of the system which satisfy the condition $\|\Delta(\bullet)(\tau)\| \leq \delta$ ( $\delta$ being a positive constant) and $u(\tau)$ is the nonlinear controller. $y$ is measurable output. $\widetilde{\omega}(\tau)=\left(\widetilde{\omega}_{1}(\tau), \ldots, \widetilde{\omega}_{n}(\tau)\right)^{T}$ is $n$-dimensional Brownian motion defined on complete probability space $(\mho, \mathscr{F}, \mathscr{P})$ with a natural filtration $\left\{\mathscr{F}_{\tau}\right\}_{\tau \geq 0}$ generated by $\{\widetilde{\omega}(s): 0 \leq s \leq \tau\}$, where $\mho$ is associated with the canonical space generated by $\{\widetilde{\omega}(\tau)\}$ with the probability measure $\mathscr{P} . \sigma: R_{+} \times R^{n} \times R^{n} \rightarrow R^{n \times n}$ is the noise intensity matrix.

Define the synchronization error vector as

$$
\begin{aligned}
e(\tau) & =x(\tau)-\widehat{x}(\tau), \\
y_{e}(\tau) & =y-\tilde{y}(\tau) .
\end{aligned}
$$

So the error dynamical system is

$$
\begin{aligned}
& d e(\tau)=[(A+F) e(\tau)-\Delta F(\tau) \hat{x}(\tau)+\Delta A(\tau) x(\tau) \\
& \quad+g(x(\tau), \widehat{x}(\tau))+\Delta B(\tau)-u(\tau)] d \tau+\sigma(t, e(\tau)) d \widetilde{\omega}(\tau),
\end{aligned}
$$

where

$$
\begin{gathered}
g(x(\tau), \widehat{x}(\tau))=D \varphi(x(\tau))-G f(\hat{x}(\tau))-F x(\tau) \\
+A \widehat{x}(\tau)+B .
\end{gathered}
$$


It is clear that the synchronization problem can be transformed to be the equivalent problem of stabilizing the error system (6).

Definition 1. Systems (3) and (4) are said to be globally stable in mean squares if for any given initial condition the following equality is verified:

$$
\lim _{\tau \rightarrow \infty} E\|e(\tau)\|^{2}=0
$$

where $E[\cdot]$ is the mathematical expectation.

Assumption 2. Let us define $h(\tau)=\Delta A(\tau)-\Delta F(\tau)$ and assume $h(\tau) \in L_{2}$; that is, $\int_{0}^{\infty} h^{T}(\tau) h(\tau) d \tau \leq \infty$.

Given a scalar $\gamma>0$, the error system is said to be robustly stochastically stable with noise attenuation $\gamma$ if it is robustly stochastically stable and under zero initial conditions:

$$
\left\|y_{e}(\tau)\right\|_{E_{2}}<\gamma\|n(\tau, h)\|_{2}
$$

For nonzero $n(\tau) \in L_{2}[0, \infty)$,

$$
\left\|y_{e}(\tau)\right\|_{E_{2}}=\left(E\left\{\int_{0}^{\infty}\left\|y_{e}(\tau)\right\|^{2} d \tau\right\}\right)^{1 / 2}
$$

In this paper, the following performance index for a prescribed $\gamma>0$ is considered:

$$
J_{e}(\tau)=E\left\{\int_{0}^{\infty}\left(y_{e}^{T}(\tau) y_{e}(\tau)-\gamma^{2} n^{T}(\tau) n(\tau)\right) d \tau\right\} .
$$

The problem to be tackled is formulated as follows. For the stochastic systems (3) and (4) and a given scalar $\gamma>0$, we like to design an adaptive feedback controller $K$ such that the resulting master-slave trajectories robustly stochastically synchronize with noise attenuation level $\gamma$ which is the disturbance attenuation level [26] defined by

$$
\gamma=\frac{E \sqrt{\int_{0}^{\infty} y_{e}^{T}(\tau) y_{e}(\tau) d \tau}}{\sqrt{\int_{0}^{\infty} n^{T}(\tau) n(\tau) d \tau}}
$$

The control requirement is that the response of error to the disturbances with the stabilization controller should be brought to less than $\gamma$ which is the suboptimal $H_{\infty}$ limits. For the standard $H_{\infty}$ problem, a controller exists if and only if a unique positive definite solution to the two Riccati equations exists [27]. State space formulas can be derived for all controllers such that the $H_{\infty}$-norm of the closed loop transfer function is less than $\gamma$.

Assumption 3. Assume that the noise intensity function matrix $\sigma: R^{+} \times R^{n} \rightarrow R^{n \times n}$ is uniformly Lipschitz continuous in terms of the norm induced by the trace inner product on the matrices as follows:

$$
\operatorname{trace}\left[\sigma^{T}(t, v) \sigma(t, v)\right] \leq v^{T} J^{T} J v
$$

for all $v \in R^{n}$. J is a real positive $n \times n$ matrix.
Definition 4. There exists continuous function $V \in C^{1,2}\left(R^{+} \times\right.$ $\left.R^{n} ; R^{+}\right)$such that an operator $\mathfrak{J} V$ from $R^{+} \times R^{n}$ along the trajectory of the error system (6) is defined as

$$
\begin{aligned}
\Im V(\tau, e)= & V_{\tau}(\tau, e)+V_{e}(\tau, e) f(\tau, e) \\
& +\left(\frac{1}{2}\right) \operatorname{trace}\left[\sigma^{T}(\tau, e) V_{e e} \sigma(\tau, e)\right],
\end{aligned}
$$

where

$$
\begin{aligned}
& V_{\tau}(\tau, e)=\frac{\partial V(\tau, e(\tau))}{\partial \tau} \\
& V_{e}(\tau, e)=\left(\frac{\partial V(\tau, e(\tau))}{\partial e_{1}}, \ldots, \frac{\partial V(\tau, e(\tau))}{\partial e_{n}}\right) \\
& V_{e e}(\tau, e)=\left(\frac{\partial^{2} V(\tau, e(\tau))}{\partial e_{i} \partial e_{j}}\right)_{n \times n} .
\end{aligned}
$$

Definition 5. Systems (3) and (4) are said to be exponentially synchronized in mean square if there exist constants $\hbar>0$ and $\lambda>0$, such that

$$
E\|e(\tau)\|^{2} \leq \lambda \exp (-\hbar \tau)
$$

with $\tau>0$ and $\hbar$ is called the decay rate of exponential synchronization.

Lemma 6. Suppose two continuous functions $f_{\mathrm{Q}} \in\left(R^{+} \times\right.$ $R \rightarrow R)$ and $\pi(\tau, x(\tau))$ a nonzero decreasing function such that $f_{\mathrm{Q}}(\tau, x(\tau))=-x^{T}(\tau) Q x(\tau)+x^{T} \pi(\tau, x(\tau))$ and then there exists a suitable positive constant $\eta^{\prime}$ satisfying the following inequality:

$$
f_{\mathrm{Q}+\eta^{\prime} I}(\tau, x(\tau)) \leq-\eta^{\prime} x^{T}(\tau) x(\tau) .
$$

\section{Main Results}

Assumption 7. We can choose the controller in this following form:

$$
u(\tau)=\frac{1}{2} K L(y-C \widehat{x}(\tau))
$$

where $L$ is the matrix with appropriate dimension and $K$ is the estimated feedback gain which is updated according to the following adaptation algorithm:

$$
\dot{K}=\rho\|y-C \widehat{x}(\tau)\|^{2},
$$

where $\rho$ is a positive constant to be specified by the designer.

The control problem with a feedback (18) is to find an admissible internally stabilizing control $K$ which would be attenuating disturbances such that the norm of the stable closed loop system from the disturbances to the controlled outputs is less than $\gamma$ ( $\gamma$ is equal to 1 for optimal and slightly greater than 1 for suboptimal control). The control objective is stated in the mathematical form in (9). One advantage of this type of controller is that it can be easily constructed through time varying resistors, capacitors, or operational amplifiers and their combinations or using a digital signal processor together with the appropriate converters. 
Assumption 8. There exist two known positive functions $\rho(x(\tau), \tau)$ and $\rho(\widehat{x}(\tau), \tau)$ such that $g(x(\tau), \widehat{x}(\tau))=M(\rho(x$, $\tau)-\rho(\widehat{x}, \tau))$. We assume the following conditions:

(1) For bounded $x$ and $\widehat{x}, \rho(x, \tau)-\rho(\widehat{x}, \tau)$ is also bounded.

(2) The function $g(x, \widehat{x})$ enters the closed ball $\Lambda$ and remains there with

$$
\begin{aligned}
\Lambda & =\left\{(x, \widehat{x}) \in R^{n \times n} \text { and } k_{0} \in R /\|g(x, \widehat{x})\|\right. \\
& \left.=M\|\rho(x, \tau)-\rho(\widehat{x}, \tau)\| \leq M k_{0}\|x-\widehat{x}\|\right\} .
\end{aligned}
$$

$M$ and $k_{0}$ are positive constants.

Assumption 9. The "robust performant" gain scheduled system is assumed to be bounded in response to certain added inputs. Moreover, the performance level must not degrade discontinuously for arbitrarily small errors in controller (18).

Lemma 10 (Gronwall inequality (see [28])). Let us consider three continuous positive functions $\varphi, \phi$, and $y$ on $\left[0, T^{\prime}\right]$ satisfying the inequality $\forall \tau \in\left[0, T^{\prime}\right], y(\tau) \leq \varphi(\tau)+$ $\int_{0}^{\tau} \phi(\tau) y(s) d s$, and then $\forall t \in\left[0, T^{\prime}\right], y(\tau) \leq \varphi(\tau)+$ $\int_{0}^{\tau} \varphi(s) \phi(s) \exp \left(\int_{s}^{\tau} \phi(u) d u\right) d s$.

Lemma 11. For two integrable functions $V \in C^{1,2}\left(R^{+} \times R^{n} ; R^{+}\right)$ and $e(\tau) \in R^{3}$, there exist two positive constants $\mu_{1}$ and $\mu_{2}$ such that

$$
\mu_{1} E\|e(\tau)\|^{2} \leq E V(\tau) \leq \mu_{2} E\|e(\tau)\|^{2} .
$$

Lemma 12. For any matrices $Q_{1} \in R^{n \times m}, Q_{2} \in R^{n \times m}, \Theta=$ $\Theta^{T}>0$, and $\Theta \in R^{n \times n}$ we have the following inequalities:

$$
Q_{1}{ }^{T} Q_{2}+Q_{2}{ }^{T} Q_{1} \leq Q_{1}{ }^{T} \Theta Q_{1}+Q_{2}{ }^{T} \Theta^{-1} Q_{2} \text {. }
$$

Assumption 13. We can choose a constant matrix $L \in R^{n \times n}$ and two positive definite matrices $P=P^{T}>0$ and $Q=Q^{T}$ such that

$$
\begin{aligned}
& (A+F-L C)^{T} P+P(A+F-L C)+2 P L C+\Omega^{2} P^{2} \\
& \quad+\sigma I=-Q, \\
& L^{T} P=C,
\end{aligned}
$$

where

$$
\begin{aligned}
\Omega^{2} & =\eta+\gamma^{-2} d^{2}+\delta_{1}+\alpha_{1} M, \\
\sigma & =\eta^{-1} \delta^{2}+\delta_{1}^{-1} \delta^{2}+\alpha_{1}^{-1} k_{0}^{2} M .
\end{aligned}
$$

Theorem 14. System (3) exponentially synchronizes system (4) under controller (18) in mean square.

Proof. The closed loop error system with control (18) can be written in the following form:

$$
\begin{aligned}
& d e(\tau)=[(A+F) e(\tau)-\Delta F(\tau) \hat{x}(\tau)+\Delta A(\tau) x(\tau) \\
& \left.\quad+g(x(\tau), \widehat{x}(\tau))+\Delta B(\tau)-\frac{1}{2} K L(\tilde{y}-C \widehat{x}(\tau))\right] d \tau \\
& \quad+\sigma(t, e(\tau)) d \widetilde{\omega}(\tau) .
\end{aligned}
$$

Consider the Lyapunov function:

$$
V(\tau)=e^{T}(\tau) P e(\tau)+\frac{1}{2 \rho}\left(K(\tau)-K_{0}\right)^{2},
$$

where $K_{0}$ is a constant to be defined later.

Relation (25) yields by differentiating $V$ with respect to time $\tau$

$$
\begin{aligned}
& \mathfrak{J} V(\tau)=\dot{e}^{T}(\tau) P e(\tau)+e^{T}(\tau) P \dot{e}(\tau)+\frac{1}{\rho}(K(\tau) \\
& \left.-K_{0}\right) \dot{K}=[(A+F-L C) e(\tau)+\Delta A(\tau) e(\tau) \\
& -\Delta F \hat{x}(\tau)+\Delta B(\tau)]^{T} P e(\tau)+e^{T}(\tau) \\
& \cdot P[(A+F-L C) e(\tau) \\
& +\Delta A(\tau) x(\tau)-\Delta F \hat{x}(\tau)+\Delta B(\tau)]^{T}+2 \bar{x}^{T} P L C e \\
& -K e^{T} P L C \bar{x}+2 e^{T} P g(x, \widehat{x})+\frac{1}{\rho}\left(K(\tau)-K_{0}\right) \dot{K} \\
& +\operatorname{trace}\left[\sigma^{T}(\tau, e(\tau)) P \sigma(\tau, e(\tau))\right] \\
& =e^{T}\left((A+F-L C)^{T} P+P(A+F-L C)\right) e(\tau) \\
& +2 e^{T}(\tau) P(\Delta A(\tau) x(\tau)-\Delta F(\tau) \widehat{x}(\tau))+2 e^{T}(\tau) \\
& \text { - } P \Delta B(\tau)+2 e^{T} P L C e-K e^{T} P L C e+2 e^{T} P g(x, \widehat{x}) \\
& +\frac{1}{\rho}\left(K(\tau)-K_{0}\right) \dot{K} \\
& +\operatorname{trace}\left[\sigma^{T}(\tau, e(\tau)) P \sigma(\tau, e(\tau))\right] \\
& =e^{T}\left((A+F-L C)^{T} P+P(A+F-L C)\right) e(\tau) \\
& +2 e^{T}(\tau) P \Delta F e(\tau)+2 e^{T}(\tau) P(\Delta A(\tau)-\Delta F(\tau)) \\
& \cdot e(\tau)+2 e^{T}(\tau) P \Delta B(\tau)+2 e^{T} P L C e-K e^{T} P L C e \\
& +2 e^{T} \operatorname{Pg}(x, \widehat{x})+\frac{1}{\rho}\left(K-K_{0}\right) \dot{K} \\
& +\operatorname{trace}\left[\sigma^{T}(\tau, e(\tau)) P \sigma(\tau, e(\tau))\right] .
\end{aligned}
$$

It is clear from Lemma 12 that

$$
\begin{aligned}
& 2 e^{T} P \Delta B(\tau) \leq \delta_{1} e(\tau)^{T} P^{2} e(\tau)+\delta_{1}^{-1} \delta^{2} e(\tau)^{T} e(\tau), \\
& 2 e^{T}(\tau) P \Delta F \bar{x}(\tau) \\
& \quad \leq \eta e^{T}(\tau) P^{2} e(\tau)+\eta^{-1} \delta^{2} e^{T}(\tau) e(\tau), \\
& 2 e^{T}(\tau) P(\Delta A-\Delta F) e(\tau) \\
& \quad \leq \gamma^{-2} d^{2} e^{T}(\tau) P^{2} e(\tau)+2 \gamma^{2} e^{T}(\tau) P h^{T} h, \\
& 2 e^{T} P g(x, \bar{x}) \\
& \quad \leq \alpha_{1} M e^{T}(\tau) P^{2} e(\tau)+\alpha_{1}^{-1} k_{0}^{2} M e^{T}(\tau) e(\tau) .
\end{aligned}
$$


For any time $\tau \geq 0$, the system state is bounded (as the system is chaotic); hence we may write $\|x(\tau)\| \leq d$. Here $d, \eta, \delta_{1}, \alpha_{1}$, and $\gamma$ are positive constants.

Relation (28) leads to

$$
\begin{aligned}
\Im & (\tau) \leq e^{T}(\tau)\left((A+F-L C)^{T} P+P(A+F-L C)\right) \\
& e(\tau)+\eta e^{T}(\tau) P^{2} e(\tau)+\eta^{-1} \delta^{2} e^{T}(\tau) e(\tau) \\
& +\gamma^{-2} d^{2} e^{T}(\tau) P^{2} e(\tau)+2 \gamma^{2} e^{T}(\tau) P h^{T}(\tau) h(\tau) \\
& +\delta_{1}^{-1} \delta^{2} e^{T}(\tau) e(\tau)+\delta_{1} e^{T}(\tau) P^{2} e(\tau)+2 \bar{x}^{T} P L C e \\
& -J^{T} J+K e^{T} P D C e+\alpha_{1} M e^{T}(\tau) P^{2} e(\tau) \\
& +\alpha_{1}^{-1} k_{0}^{2} M e^{T}(\tau) e(\tau)+\frac{1}{\rho}\left(K-K_{0}\right) \dot{K} \leq e^{T}(\tau) \\
& +\left[(A+F-L C)^{T} P\right. \\
& +P(A+F-L C)+\eta P^{2}+\eta^{-1} \delta^{2} I+\gamma^{-2} d^{2} P^{2} \\
& \left.+\delta_{1}^{-1} \delta^{2} I+\delta_{1} P^{2}\right] e(\tau)+2 \gamma^{2} e^{T}(\tau) P h^{T}(\tau) h(\tau) \\
+ & 2 e^{T} P L C e-K e^{T} P L C e+\alpha_{1} M e^{T}(\tau) P^{2} e(\tau) \\
& \left.+2 \gamma^{2} e^{T}(\tau) P h^{T}(\tau) h(\tau)-K_{0}\right) \dot{K} . \\
& +2 P L C+\left(\eta+\nu^{T} P e \|^{2}+\frac{1}{\rho}(K\right. \\
+ & \alpha_{1}^{-1} k_{0}^{2} M e^{T}(\tau) e(\tau)+e^{T}(\tau) J^{T} J e(\tau)+\frac{1}{\rho}(K \\
& \left.-K_{0}\right) \dot{K} \leq e^{T}\left(\left(A+\delta_{1}^{-1} \delta^{2}+\alpha_{1}^{-1} k_{0}^{2} M\right) I\right) P^{2} \\
& \left.+J^{T} J\right) e
\end{aligned}
$$

Let one recall relations (23):

$$
\begin{aligned}
& \Omega^{2}=\eta+\gamma^{-2} d^{2}+\delta_{1}+\alpha_{1} M, \\
& \sigma=\eta^{-1} \delta^{2}+\delta_{1}^{-1} \delta^{2}+\alpha_{1}^{-1} k_{0}^{2} M
\end{aligned}
$$

and let $2 \gamma^{2}=K_{0}$.

Remark 1 . There exist a lot of papers that study $H_{-\infty}$ synchronization problems of master-slave chaotic systems, in which the choice of the performance index which satisfies condition (9) remains a hard task in practice and leads sometimes to the poor performance of the control. Our method does not need the arbitrary choice of this quantity. Besides, the control objective is obtained by the selection of the initial value of the feedback gain which enables approaching the synchronization. This partly shows the less conservativeness of our control strategy.
Using inequality (31), we obtain

$$
\begin{aligned}
& \Im V(\tau) \leq e^{T}(\tau)\left((A+F-L C)^{T} P+P(A+F-L C)\right. \\
& \left.\quad+2 P L C+\Omega^{2} P^{2}+J^{T} J+\sigma I\right) e(\tau)+2 \gamma^{2} e^{T}(\tau) \\
& \quad \cdot P\left(h^{T}(\tau) h(\tau)-\frac{\dot{K}}{\rho}\right) .
\end{aligned}
$$

Finally, by letting

$$
\begin{aligned}
& \omega^{T}(\tau) \omega(\tau)=\gamma^{2}\left(h^{T}(\tau) h(\tau)-\frac{\dot{K}}{\rho}\right) \\
& (A+F-L C)^{T} P+P(A+F-L C)+2 P L C+\Omega^{2} P^{2} \\
& \quad+J^{T} J+\sigma I=-Q
\end{aligned}
$$

one obtains

$$
\mathfrak{J} V(\tau) \leq-e^{T} Q e+2 e^{T}(\tau) P \omega^{T}(\tau) \omega(\tau) .
$$

Then, if the inequality $-e^{T} \mathrm{Q} e+2 e^{T}(\tau) P \omega^{T}(\tau) \omega(\tau)<0$ holds, we have

$$
\mathfrak{\Im} V(\tau) \leq\left[\begin{array}{l}
e(\tau) \\
\omega(\tau)
\end{array}\right]^{T}\left[\begin{array}{cc}
-Q & P \\
P & 0
\end{array}\right]\left[\begin{array}{l}
e(\tau) \\
\omega(\tau)
\end{array}\right] \leq 0 .
$$

For all nonzero $\omega(\tau) \in L_{2}[0, \infty)$, under zero initial conditions, we have $E\{V(0)\}=V_{0}$ and $E\{V(\tau)\} \geq 0$. Integrating both sides of (35) from 0 to $\tau>0$ and taking the expectation, we have

$$
E\{V(\tau)\}=E\left\{\int_{0}^{\tau} \mathfrak{\Im} V(t)\right\} d t
$$

We derive the cost function as

$$
\begin{aligned}
J_{e} & =E\left\{\int _ { 0 } ^ { \tau } \left[y_{e}^{T}(t) y_{e}(t)-\gamma^{2} \varpi^{T}(t) \omega(t)\right.\right. \\
& +\mathfrak{\Im} V(t)] d t\}-E\{V(t)\} \leq E\left\{\int _ { 0 } ^ { \tau } \left[y_{e}^{T}(t) y_{e}(t)\right.\right. \\
& \left.\left.-\gamma^{2} \omega^{T}(t) \omega(t)+\mathfrak{J} V(t)\right] d t\right\} .
\end{aligned}
$$

Substituting (35) into (37) yields

$$
J_{e}(e(\tau), \omega(\tau)) \leq E\left\{\left[\begin{array}{l}
e(\tau) \\
\omega(\tau)
\end{array}\right]^{T} \Psi\left[\begin{array}{l}
e(\tau) \\
\omega(\tau)
\end{array}\right]\right\}
$$

where

$$
\begin{aligned}
& \Psi=\left[\begin{array}{cc}
-Q+\bar{C} & P \\
P & -\gamma^{2} I
\end{array}\right], \\
& \bar{C}=\left[\begin{array}{cc}
C^{T} C & 0 \\
0 & 0
\end{array}\right] .
\end{aligned}
$$


For a positive constant $\lambda$, if the following condition holds:

$$
\widehat{\Psi}=\Psi+\left[\begin{array}{cc}
\lambda P & 0 \\
0 & 0
\end{array}\right]=\left[\begin{array}{cc}
-Q+C+\lambda P & P \\
P & -\gamma^{2} I
\end{array}\right]<0,
$$

then we have

$$
\begin{gathered}
J_{e}(e(\tau), \omega(\tau)) \leq E\left\{\left[\begin{array}{l}
e(\tau) \\
\omega(\tau)
\end{array}\right]^{T} \Psi\left[\begin{array}{l}
e(\tau) \\
\omega(\tau)
\end{array}\right]\right\} \\
=E\left\{\left[\begin{array}{l}
e(\tau) \\
\omega(\tau)
\end{array}\right]^{T}\left(\widehat{\Psi}-\left[\begin{array}{ll}
\lambda P & 0 \\
0 & 0
\end{array}\right]\right)\left[\begin{array}{l}
e(\tau) \\
\omega(\tau)
\end{array}\right]\right\} \\
<-E\left\{\left[\begin{array}{l}
e(\tau) \\
\omega(\tau)
\end{array}\right]^{T}\left[\begin{array}{ll}
\lambda P & 0 \\
0 & 0
\end{array}\right]\left[\begin{array}{l}
e(\tau) \\
\omega(\tau)
\end{array}\right]\right\}
\end{gathered}
$$

which leads to the following conclusion: $J_{e}(e(\tau), \omega(\tau))<0$. This implies that the error system (20) via control inputs $u(\tau)$ with the update law (19) is asymptotically stable. Then, (9) follows immediately from (37) and the proof of Definition 1 is completed.

From Lemma 6, we get

$$
\Im V(\tau) \leq-\eta^{\prime} e^{T} e .
$$

It follows from Ito's formula that

$$
\begin{gathered}
E V(\tau, e(\tau))-E V(0, e(0))=\int_{0}^{\tau} E \mathfrak{I} V \\
\leq-\eta^{\prime} \int_{0}^{\tau} E\left(e(s)^{T} e(s)\right) d s, \quad \tau>0 .
\end{gathered}
$$

From Lemma 11, (43) becomes

$$
\begin{aligned}
\lambda_{\min }(P) E\|e(\tau)\|^{2} & \leq E V(\tau, e(\tau)) \\
& \leq E V(0, e(0)) \\
& -\eta^{\prime} \int_{0}^{\tau} E\|e(s)\|^{2} d s .
\end{aligned}
$$

Using Gronwall's inequality (Lemma 10), we have

$$
E\|e(\tau)\|^{2} \leq \widetilde{\varepsilon} \exp (-\widehat{\varepsilon} \tau),
$$

where $\widetilde{\varepsilon}=E V(0, e(0)) / \lambda_{\min }(P)$ and $\widehat{\varepsilon}=\eta^{\prime} / \lambda_{\min }(P)$.

Relation (45) guarantees the exponential synchronization. This completes the proof.

Remark 2. It is important to show that the trajectories of the slave system are bounded under the feedback in the presence of perturbations:

$$
\sup _{\tau \geq 0}\left|\widehat{x}_{1,3}\right| \leq \pi
$$

(R-1) Since the systems are assumed to operate in chaotic mode without feedback, their trajectories converge to compact invariant set. Let one assume that $\xi$ is the spectral radius of the perturbation on the system dynamics. We set $r+\xi>0$, and let the closed ball $\bar{B}_{r+\xi}$ strictly contains such a compact set (since the chaotic trajectories are bounded, we assume that they are contained in $\bar{B}_{r+\xi}$ ); let $\infty>T \geq 0$ be the smallest number such that $e(\tau) \in \bar{B}_{r+\xi} \forall \tau \geq T$. The previous development shows that $V$ is positive definite Lyapunov function with a negative definite derivative for any values of the states contained in a compact set. Hence, there always exist control gains such that the system under feedback is forward complete.

(R-2) It may be shown that the system under control is forward complete; that is, if there exists a set of initial conditions and gains such that, together, they generate solutions that tend to infinity, these solutions may unboundedly grow only in infinite time. From this, it follows that for each $\theta>0$ and $T>0$, there exists $M(\theta, T)$ such that $|e(0)| \leq \theta+\xi \Rightarrow$ $|e(\tau)| \leq M(\theta+\xi, T), \forall \tau \in[0, T]$, where $M$ is in general a nondecreasing function of its arguments. We assume that $T$ is the synchronization time since, by assumption, the system operates in open loop for all $\tau \in[0, T]$ for any $T>0,|e(\tau)| \leq \max \{M(0+$ $\xi, T), r+\xi\}$ for all $\tau \in[0, T]$ for any $T>0$. That is, the solutions are bounded. Note that the bound $\max \{M(\theta+\xi, T), r+\xi\}$ is independent of the gains, and for $T \geq T^{*}$, we can safely assume that $\max \{M(0+$ $\xi, T), r+\xi\}=r+\xi$ and $\pi$ depends only on $r$; hence, (R-2) of the proposition follows. If $T$ and $\theta_{\xi}\left(\theta_{\xi}=\right.$ $\max (\theta+\xi))$ are given and $\max \{M(\theta+\xi, T), r+\xi\}=M$, then $\pi$ depends on $T$ and $\theta_{\xi}$ and so does $K$; hence, (R$1)$ of the proposition follows. In either case, $K$ does not depend on the initial conditions $e(0)$, nor is it required that $|e(0)| \leq r+\xi$.

\section{Simulation Investigation}

In this section, we will provide simulation results for a system where the master oscillator is the perturbed modified Colpitts oscillators and the observer is a perturbed and stochastic Chua system.

From (1), the modified Colpitts systems whose dimensionless equations are given in [29] is represented by the values as follows:

$$
\begin{aligned}
& x=\left[\begin{array}{l}
x_{1} \\
x_{2} \\
x_{3}
\end{array}\right], \\
& A=\left[\begin{array}{lll}
0 & 0 & 1 \\
0 & -b_{0} & 1 \\
-1 & -1 & -c_{11}
\end{array}\right], \\
& B=\left[\begin{array}{c}
0 \\
-b_{0} \\
1
\end{array}\right],
\end{aligned}
$$



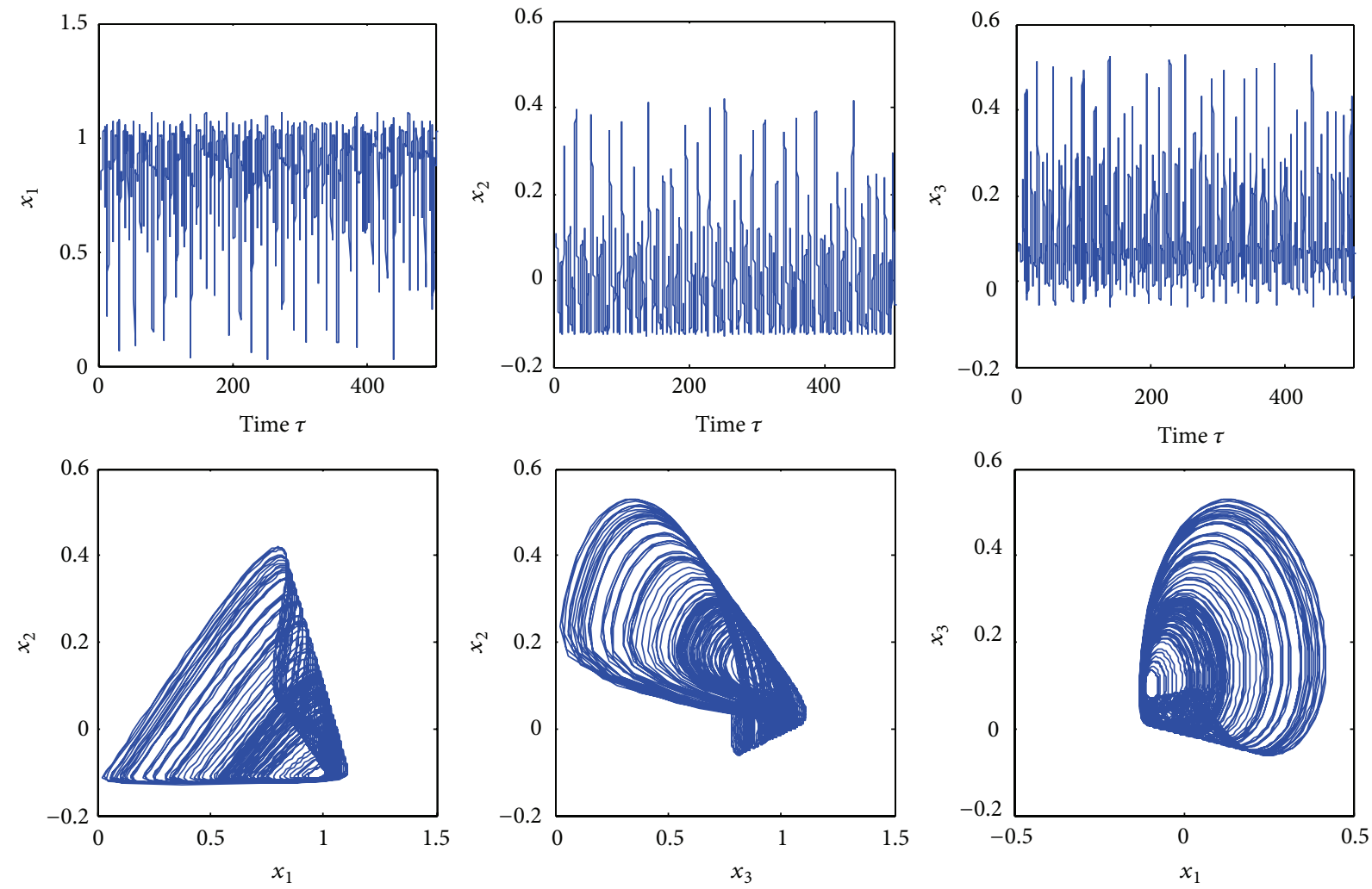

FIgURE 1: Time history and phase portraits of modified Colpitts oscillator.

$$
\begin{gathered}
D=\left[\begin{array}{ccc}
-1 & 0 & 0 \\
0 & 0 & 0 \\
0 & 0 & 0
\end{array}\right], \\
\varphi(x)=\left[\begin{array}{c}
\varphi\left(x_{2}, x_{3}\right) \\
0 \\
0
\end{array}\right] .
\end{gathered}
$$

The slave based on the observer method is the Chua's circuit and can be generalized in form (2) [30], where

$$
\begin{aligned}
F & =\left[\begin{array}{ccc}
-\alpha & \alpha & 0 \\
0 & -1 & 1 \\
-1 & -\beta_{1} & -\beta_{2}
\end{array}\right], \\
G & =\left[\begin{array}{ccc}
-\alpha & 0 & 0 \\
0 & 1 & 0 \\
0 & 0 & 1
\end{array}\right], \\
f(\hat{x}) & =\left[\begin{array}{c}
f\left(\hat{x}_{1}\right) \\
0 \\
0
\end{array}\right] .
\end{aligned}
$$

$\varphi\left(x_{2}, x_{3}\right)$ and $f\left(\hat{x}_{1}\right)$ are continuous-time functions defined, respectively, by $\varphi\left(x_{2}, x_{3}\right)=a_{2} \exp \left(-a x_{3}-b x_{2}\right)$ and $f\left(\hat{x}_{1}\right)=$ $b^{\prime} \hat{x}_{1}+0.5\left(a^{\prime}-b^{\prime}\right)\left(\left|\widehat{x}_{1}+1\right|-\left|\widehat{x}_{1}-1\right|\right)$ with $a^{\prime}<b^{\prime}<0$.

If the parameters are taken as $\alpha=10, \beta_{1}=15, \beta_{2}=$ $0.0385, a^{\prime}=-1.28, b^{\prime}=-0.69, a=2.251362, b=192.3, b_{0}=$
$0.1064814815, c_{11}=0.934$, and $a_{2}=8.518518 \times 10^{-11}$, the systems specified by (46) and (47) exhibit chaotic dynamics. Figures 1 and 2 give the time history and phase portrait of both systems.

Remark 3. Systems (1) and (2) are quite different to make our illustration general. Most previous studies in the literature have predominantly concentrated on standard systems such as the Lorentz, the classic Colpitts oscillator, the Chua system, the Chen system, the Lu system, or the Rössler system either in the studies of their stability analysis and periodic oscillations or in the studies of their synchronization (the general form of these dynamical systems is provided in (1)). It has been shown that the modified Colpitts oscillator presents different topology and can exhibit complicated dynamics with reference to circuits previously mentioned. If $B \neq 0$, the problem becomes complex and requires some supplementary conditions by comparison to those employed in the literature; this aspect makes the present result more general with respect to those encountered in the literature.

For convenience, we select the following matrices:

$$
\begin{aligned}
& C=\Lambda\left[\begin{array}{ccc}
-1 & 0 & -1 \\
0 & 0 & 0 \\
0 & 0 & 0
\end{array}\right], \\
& L=\left[\begin{array}{lll}
1 & 0 & 0 \\
0 & 1 & 0 \\
0 & 0 & 1
\end{array}\right],
\end{aligned}
$$

where $\Lambda \neq 0$. 

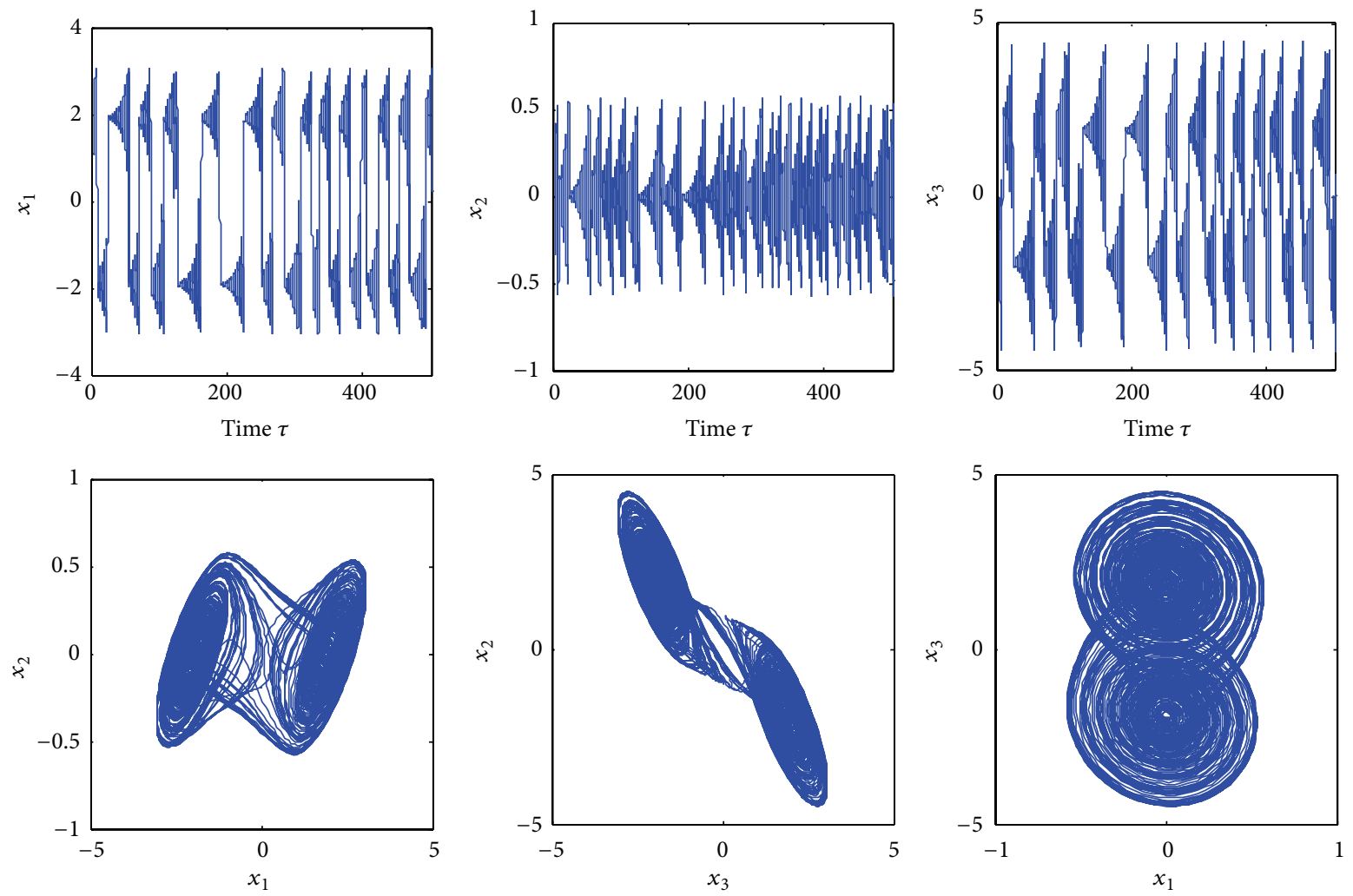

FIgURE 2: Time history and phase portraits of Chua's circuit.

If we let $\Lambda=-10^{-2}$, then the estimated feedback gain is updated according to the following adaptation algorithm:

$$
\dot{K}=\rho\left[\left(x_{1}-\widehat{x}_{1}\right)^{2}+\left(x_{3}-\widehat{x}_{3}\right)^{2}\right] .
$$

Since structural variations may take any forms in theory depending on the sources of disturbances, we illustrate the proposed control scheme with a more global example where many perturbation sources are taken into consideration [2931] under the assumption that $\|\Delta(\bullet)(\tau)\| \leq \delta$. Hence we choose an arbitrary structural variation as follows:

$$
\begin{aligned}
& \Delta A=\phi(\tau)\left[\begin{array}{ccc}
0.1 & 0 & -0.3 \\
0 & -0.5 & 0 \\
0.4 & 0 & 0.8
\end{array}\right] ; \\
& \Delta F_{0}=\phi(\tau)\left[\begin{array}{ccc}
0.8 & 0 & -2.4 \\
0 & -4 & 0 \\
3.2 & 0 & 6.4
\end{array}\right],
\end{aligned}
$$

where $\phi(\tau) \in[-1,1]$. form:

Let us select the perturbed matrix simply in the following

$$
\Delta B(\tau)=\left[\begin{array}{c}
\varepsilon_{1}(\tau) \\
\varepsilon_{2}(\tau) \\
0
\end{array}\right] .
$$

We adopt $\varepsilon_{1}(\tau)$ as an additive Gaussian noise such that $\left\|\varepsilon_{1}(\tau)\right\| \leq 1.75$ and $\varepsilon_{2}(\tau)$ is estimated as $\varepsilon_{2}(\tau)=$ $0.8 \sin (8 \tau)+0.3 \sin (12 \tau)$. The noise perturbation can be taken as $\sigma(\tau, e(\tau))=\operatorname{diag}\left(\sqrt{0.002} e_{x}(\tau), \sqrt{0.065} e_{y}(\tau), \sqrt{0.042} e_{z}(\tau)\right)$ and $\omega(\tau)$ is a 3 -dimensional Brownian motion satisfying $E\{d \omega(\tau)\}=0$ and $E\left\{[d \omega(\tau)]^{2}\right\}=d \tau$. The initial values of master system and the slave system are adopted as $\left(x_{10}, x_{20}\right.$, $\left.x_{30}\right)=\left(2 \times 10^{-5}, 2 \times 10^{-5}, 2 \times 10^{-6}\right)$ and $\left(\widehat{x}_{10}, \widehat{x}_{20}, \widehat{x}_{30}\right)=(8 \times$ $\left.10^{-3}, 6,5\right)$, respectively. In addition, we select $\rho=0.5$ and $K(0)=1.28$ which lead to $\gamma=0.80$.

Figure 3 shows the time response of the synchronization errors without the adaptive robust feedback controller $u(\tau)$ designed in (18). From these figures, one can see that the synchronization errors diverge. Figure 4 depicts the output errors with the robust adaptive feedback controller $u(\tau)$, from which we can see that the master system (3) and slave system (4) can be exponentially synchronized in mean square. From the theoretical analysis, it appears that the complexity of perturbations cannot give any information about the performance of the system. It is hard to obtain an optimal value of feedback control gain. In order to explore the synchronization behavior of the scheme, $K$ is taken as a variable of the output error (we recall that the initial value of the system variables does not affect the evolution of the feedback gain) to see how the solution of the output error evolves with the variation of the parameter. From Figure 5, it can be observed that $y_{e x}$ and $y_{e z}$ converge to zero at about $K=1.827$ which means that the synchronization can be achieved when $K>1.827$. 

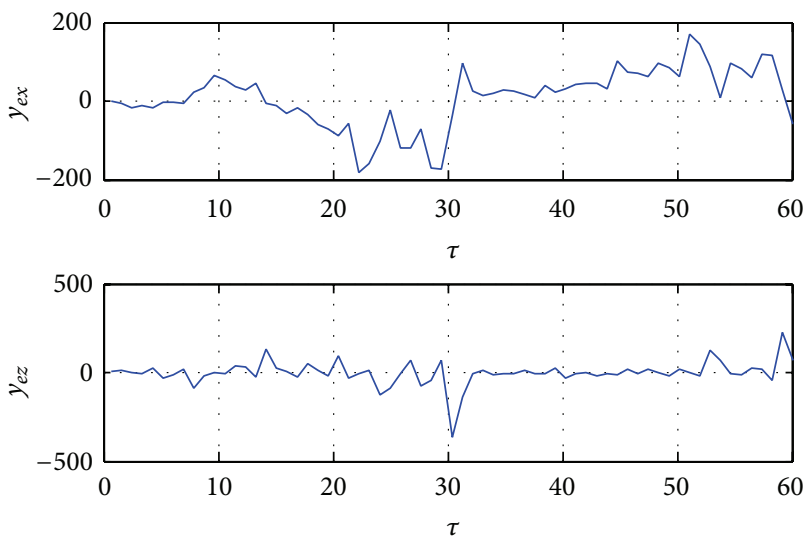

FIGURE 3: The time history of the output error with disturbances when no control input is applied.
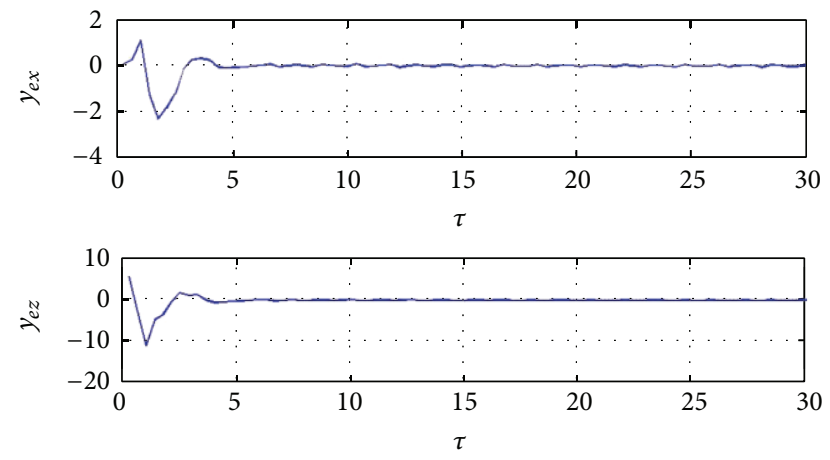

Figure 4: The time history of the output errors when the control input is applied in presence of structural perturbation.
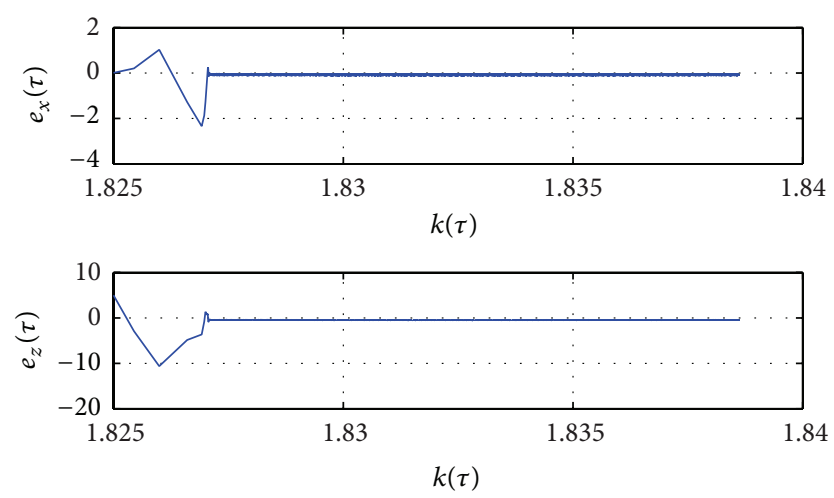

FIGURE 5: Time evolution of the estimated feedback gain $K$ in presence of structural perturbation.

\section{Conclusion}

Because the adaptive control has some interesting features such as low sensitivity to external disturbances, robustness to the plant uncertainties, and easy realization, in this paper, we use this method to realize exponential synchronization of two different uncertain chaotic systems in which the slave system is noise perturbed. By employing the Lyapunov functional method and adaptive control, several sufficient conditions have been obtained which ensure the coupled chaotic systems to be exponentially robustly synchronized in the mean square. Furthermore, the theoretical analysis is easily verified by using the standard numerical software. We have selected two perturbed systems consisting of modified Colpitts oscillator and Chua's oscillator. It was found that the controller maintains robust stable synchronization in the presence of exoteric perturbations, structural uncertainties, and noises.

\section{Conflict of Interests}

The authors declare that there is no conflict of interests regarding the publication of this paper.

\section{References}

[1] L. M. Pecora and T. L. Carroll, "Synchronization in chaotic systems," Physical Review Letters, vol. 64, no. 8, pp. 821-824, 1990.

[2] G. Chen and X. Dong, "From chaos to order. Perspective and methodologies in controlling chaotic nonlinear dynamical systems," International Journal of Bifurcation and Chaos, vol. 3, no. 6, pp. 1363-1409, 1993.

[3] E. Bai and K. Lonngen, "Synchronization of two Lorenz systems using active control," Physical Review E, vol. 8, pp. 51-59, 1997.

[4] Y. Yu and S. Zhang, "Adaptive backstepping synchronization of uncertain chaotic system," Chaos, Solitons and Fractals, vol. 21, no. 3, pp. 643-649, 2004.

[5] H. Salarieh and M. Shahrokhi, "Adaptive synchronization of two different chaotic systems with time varying unknown parameters," Chaos, Solitons and Fractals, vol. 37, no. 1, pp. 125136, 2008.

[6] K. Konishi, M. Hirai, and H. Kokame, "Sliding mode control for a class of chaotic systems," Physics Letters A, vol. 245, no. 6, pp. 511-517, 1998.

[7] J. M. Nazzal and A. N. Natsheh, "Chaos control using slidingmode theory," Chaos, Solitons \& Fractals, vol. 33, no. 2, pp. 695702, 2007.

[8] J. Alvarez-Gallegos, "Nonlinear regulation of a Lorenz system by feedback linearization techniques," Dynamics and Control, vol. 4, no. 3, pp. 277-298, 1994.

[9] A. Alasty and H. Salarieh, "Nonlinear feedback control of chaotic pendulum in presence of saturation effect," Chaos, Solitons and Fractals, vol. 31, no. 2, pp. 292-304, 2007.

[10] A. Alasty and H. Salarieh, "Controlling the chaos using fuzzy estimation of OGY and Pyragas controllers," Chaos, Solitons and Fractals, vol. 26, no. 2, pp. 379-392, 2005.

[11] H. Salarieh and M. Shahrokhi, "Adaptive synchronization of two different chaotic systems with time varying unknown parameters," Chaos, Solitons and Fractals, vol. 37, no. 1, pp. 125136, 2008.

[12] W. Yu and J. Cao, "Adaptive synchronization and lag synchronization of uncertain dynamical system with time delay based on parameter identification," Physica A, vol. 375, no. 2, pp. 467482, 2007.

[13] P. Louodop, H. B. Fotsin, and S. Bowong, "A strategy for adaptive synchronization of an electrical chaotic circuit based on nonlinear control," Physica Scripta, vol. 85, no. 2, Article ID 025002, 2012. 
[14] J. H. Park, "Adaptive controller design for modified projective synchronization of Genesio-Tesi chaotic system with uncertain parameters," Chaos, Solitons \& Fractals, vol. 34, no. 4, pp. 11541159, 2007.

[15] F. Sorrentino, G. Barlev, A. B. Cohen, and E. Ott, "The stability of adaptive synchronization of chaotic systems," Chaos, vol. 20, no. 1, Article ID 013103, 2010.

[16] H. R. Koofigar, F. Sheikholeslam, and S. Hosseinnia, "Robust adaptive synchronization for a general class of uncertain chaotic systems with application to Chua's circuit," Chaos, vol. 21, no. 4, Article ID 043134, 2011.

[17] Y.-A. Hu, H.-Y. Li, C.-P. Zhang, and L. Liu, "Robust adaptive finite-time synchronization of two different chaotic systems with parameter uncertainties," Journal of Applied Mathematics, vol. 2012, Article ID 607491, 16 pages, 2012.

[18] S. Bowong and J. J. Tewa, "Unknown inputs' adaptive observer for a class of chaotic systems with uncertainties," Mathematical and Computer Modelling, vol. 48, no. 11-12, pp. 1826-1839, 2008.

[19] S.-J. Ma, D. Dong, and J. Zheng, "Generalized synchronization of stochastic discrete chaotic system with poisson distribution coefficient," Discrete Dynamics in Nature and Society, vol. 2013, Article ID 981503, 8 pages, 2013.

[20] Z. Y. Wang and L. H. Huang, "Robust decentralized adaptive control for stochastic delayed Hopfield neural networks," Neurocomputing, vol. 74, no. 17, pp. 3695-3699, 2011.

[21] X. Ding, Y. Gao, W. Zhou, D. Tong, and H. Su, "Adaptive almost surely asymptotically synchronization for stochastic delayed neural networks with Markovian switching," Advances in Difference Equations, vol. 2013, article 211, 2013.

[22] X. Li and X. Fu, "Synchronization of chaotic delayed neural networks with impulsive and stochastic perturbations," Communications in Nonlinear Science and Numerical Simulation, vol. 16, no. 2, pp. 885-894, 2011.

[23] M. Chen and W.-H. Chen, "Robust adaptive neural network synchronization controller design for a class of time delay uncertain chaotic systems," Chaos, Solitons and Fractals, vol. 41, no. 5, pp. 2716-2724, 2009.

[24] H. Meng and W. Lili, "Robust stability and $H_{\infty}$-control of uncertain systems with impulsive perturbations," Advances in Difference Equations, vol. 2014, article 79, 2014.

[25] Y. Fang, K. Yan, and K. Li, "Robust adaptive exponential synchronization of stochastic perturbed chaotic delayed neural networks with parametric uncertainties," Mathematical Problems in Engineering, vol. 2014, Article ID 963081, 12 pages, 2014.

[26] A. Stoorvogel, The $\mathrm{H}_{\infty}$ Control Problem: A State-Space Approach, Prentice-Hall, 1992.

[27] J. C. Doyle, K. Glover, P. P. Khargonekar, and B. A. Francis, "State-space solutions to standard $\mathrm{H}_{2}$ and $H_{\infty}$ control problems," IEEE Transactions on Automatic Control, vol. 34, no. 8, pp. 831-847, 1989.

[28] B. Øksendal, Stochastic Differential Equations. An Introduction with Applications, Springer, New York, NY, USA, 2005.

[29] S. T. Kammogne and H. B. Fotsin, "Synchronization of modified Colpitts oscillators with structural perturbations," Physica Scripta, vol. 83, no. 6, Article ID 065011, 2011.

[30] L. O. Chua, "Global unfolding of Chua's circuit," IEICE Transactions on Fundamentals of Electronics, vol. 76, pp. 704-734, 1993.

[31] S. T. Kammogne, H. B. Fotsin, M. Kounchou, and P. Louodop, "A robust observer design for passivity-based synchronization of uncertain modified Colpitts oscillators and circuit simulation," Asian Journal of Science and Technologies, vol. 5, pp. 29-41, 2013. 


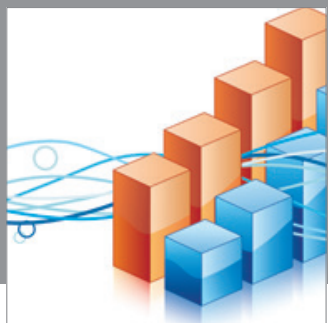

Advances in

Operations Research

mansans

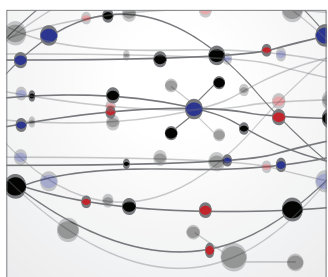

The Scientific World Journal
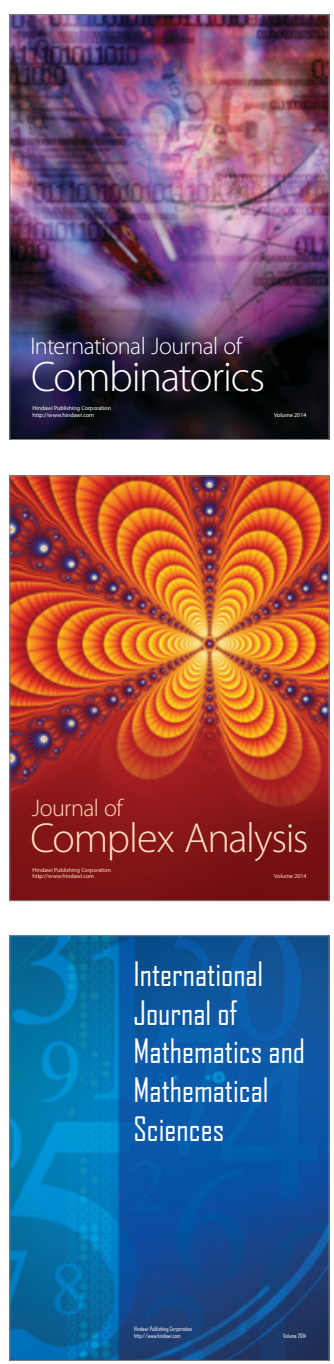
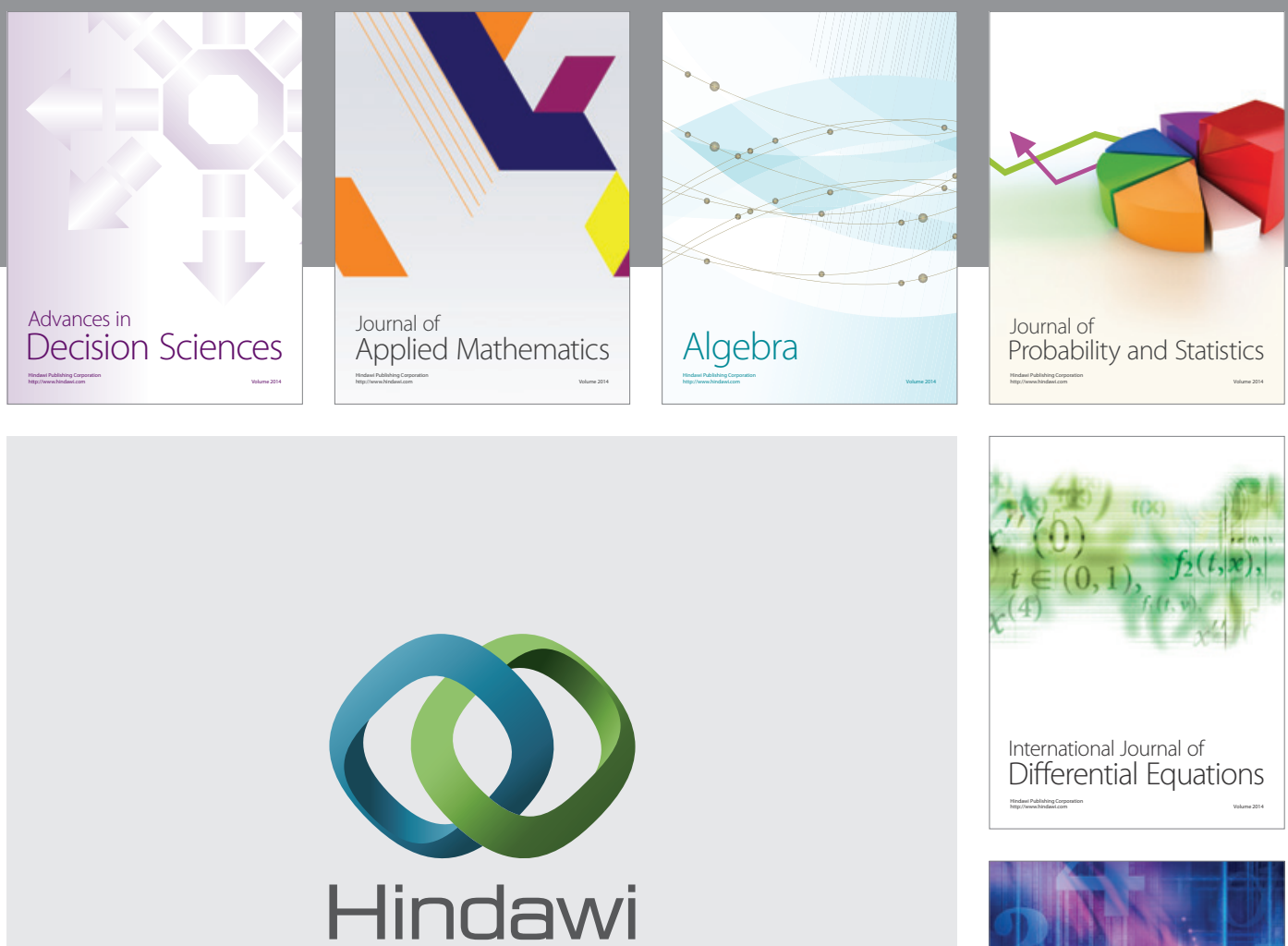

Submit your manuscripts at http://www.hindawi.com
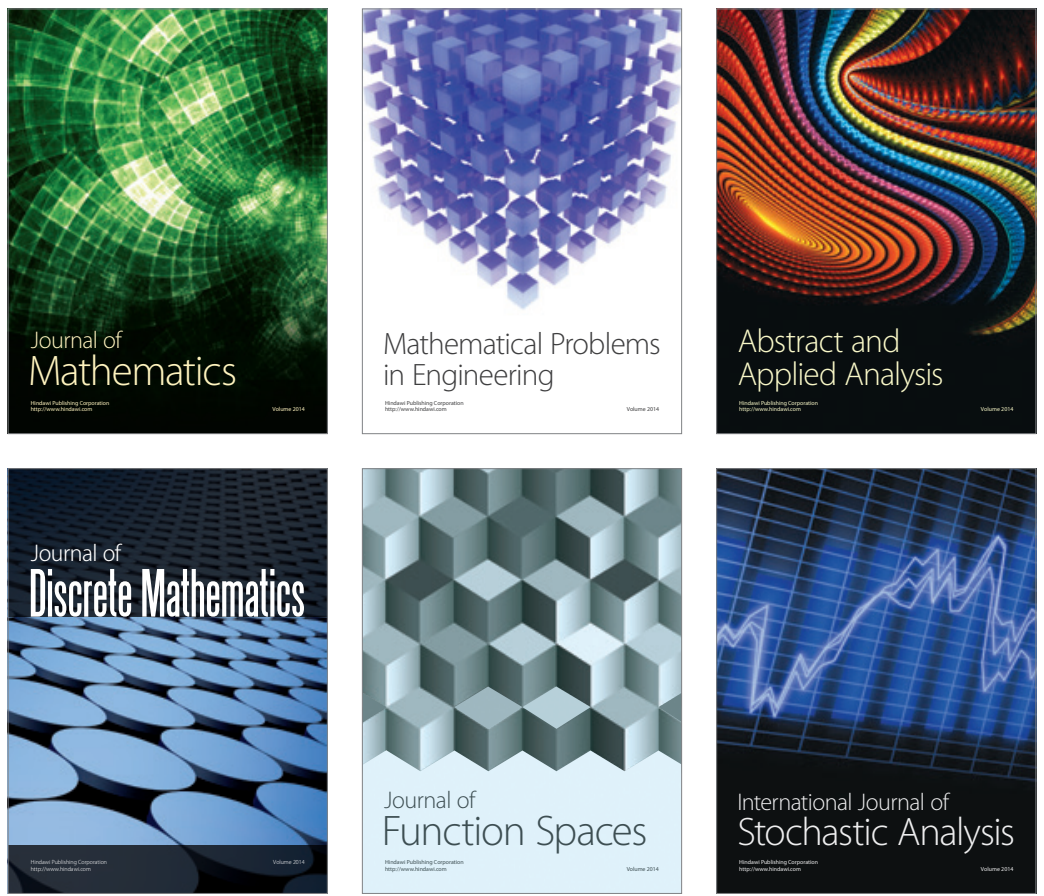

Journal of

Function Spaces

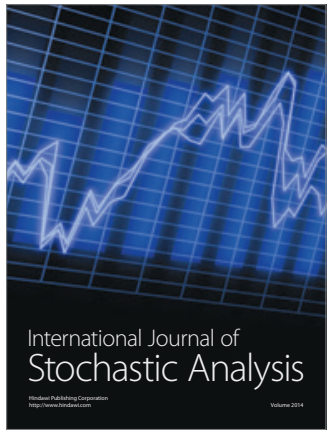

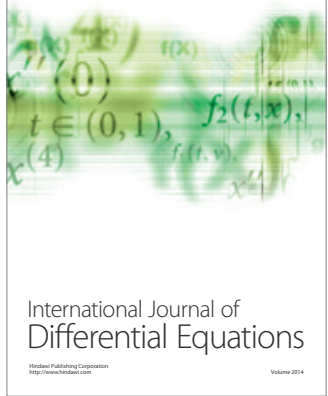
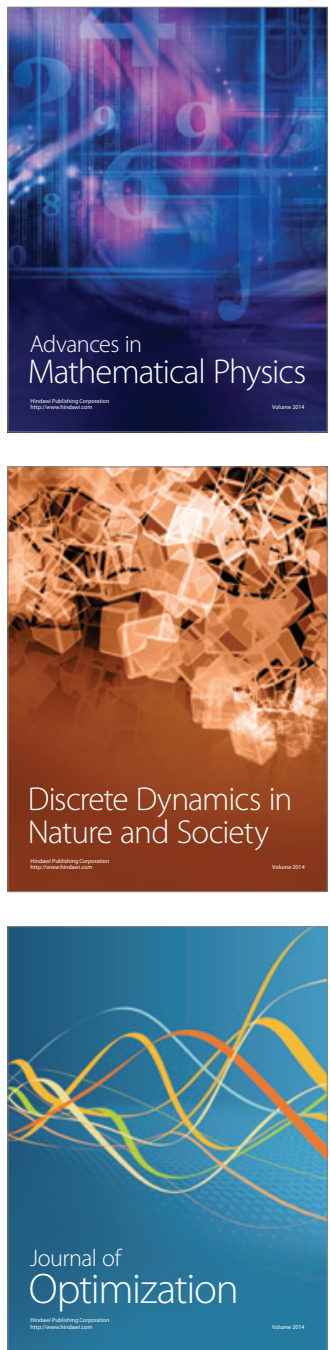